\title{
Intramyocardial Calcification with Mitral Annular Calcification
}

\author{
Yuhei Shiga, Shin-ichiro Miura, Ayumi Nakamura and Keijiro Saku
}

Key words: myocardial calcification, mitral annular calcification, echocardiogram

(Intern Med 50: 1857-1858, 2011)

(DOI: 10.2169/internalmedicine.50.5739)
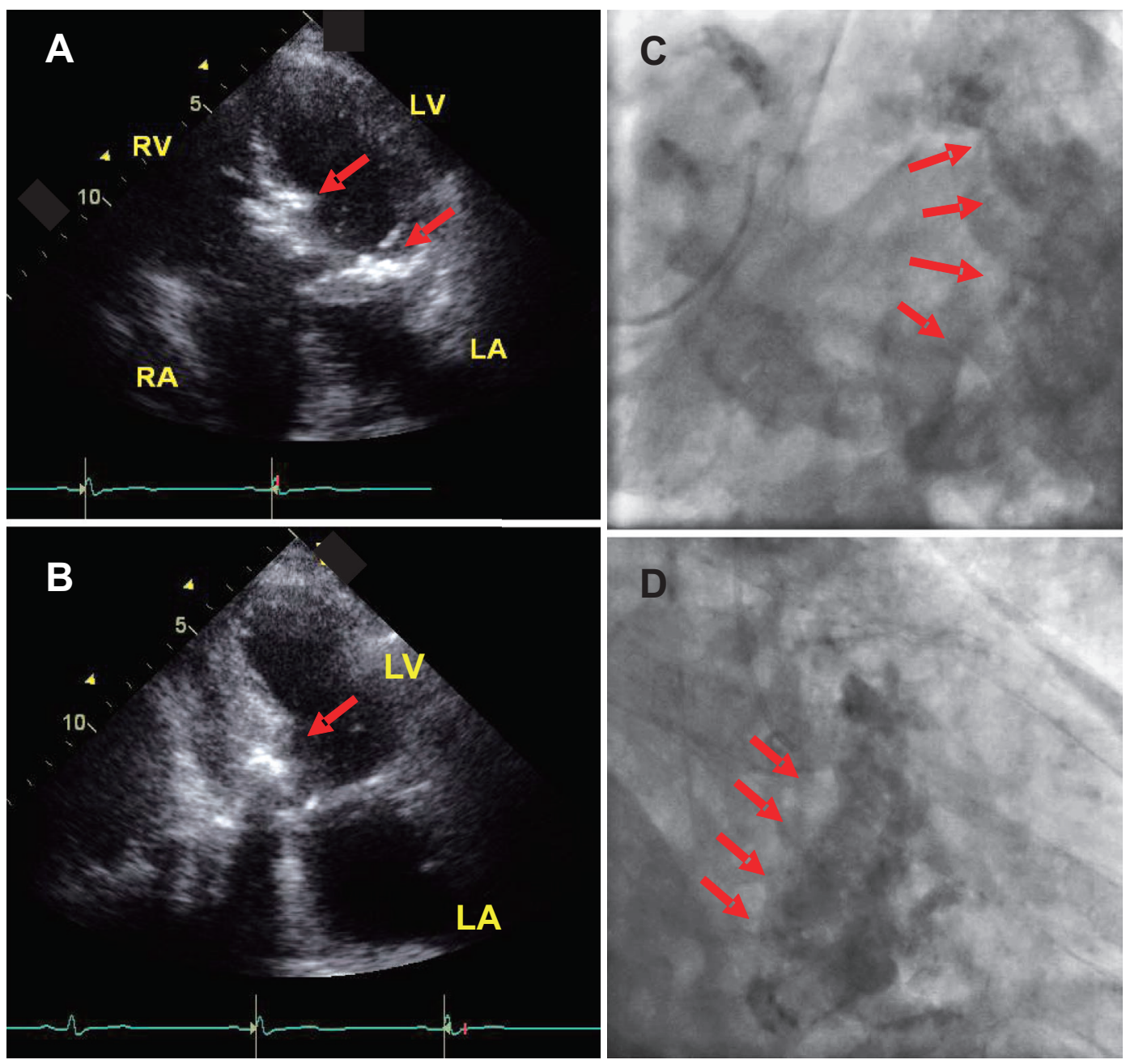

Picture.

RV: right ventricle, $R A$ : right atrium, $L V$ : left ventricle, LA: left atrium, LAO: left anterior oblique position, CAU: caudal position, RAO: right anterior oblique position

An 82-year-old man was admitted to our hospital due to atypical chest pain. He had a history of pulmonary tubercu- losis in childhood and angina pectoris for more than 4 years. Transthoracic echocardiogram showed normal left 
ventricular systolic function, left atrial dilatation, and mitral regurgitation without mitral stenosis, along with mitral annular calcification (MAC) and massive intramyocardial calcification between the atrioventricular septum and the lateral wall in the left ventricle (Picture A, B, arrow). Furthermore, cardiac catheterization to rule out ischemic heart disease also showed intramyocardial calcification (Picture C, D, arrow). No other disease that may have caused dystrophic myocardial calcification, such as severe renal insufficiency, hyperparathyroidism, or cancer, was present. A previous report described intramyocardial calcification of the ventricular septum and the lateral free wall in a 71-year-old woman with a history of childhood pulmonary tuberculosis (1). In the present case, intramyocardial calcification with MAC may also have developed secondarily from myocardial inflammation due to tuberculosis.

The authors state that they have no Conflict of Interest (COI).

\section{Reference}

1. Butz T, van Bracht M, Meissner A, et al. Mitral valve disease as well as uncommon extensive epipericardial and intramyocardial calcification secondary to massive mitral annular calcification. Eur J Echocardiogr 11: 85-88, 2010.

(C) 2011 The Japanese Society of Internal Medicine http://www.naika.or.jp/imindex.html 